\title{
Mikrochirurgische Ausbildung in Deutschland - Ergebnisse einer Umfrage unter Weiterbildungsassistenten und Weiterbildern
}

\author{
Microsurgical Training in Germany - Results of a Survey among Trainers and \\ Trainees
}

Autoren

Institute
J. Kolbenschlag ${ }^{1}$, B. Gehl' ${ }^{2}$, A. Daigeler ${ }^{1}$, T. Kremer ${ }^{3}$, C. Hirche ${ }^{3}$, P. M. Vogt ${ }^{4}$, R. Horch ${ }^{5}$, M. Lehnhardt ${ }^{1}$, U. Kneser ${ }^{3}$

Die Institutsangaben sind am Ende des Beitrags gelistet

\author{
Schlüsselwörter \\ - Weiterbildung<Sonstige \\ Aspekte \\ - Mikrochirurgie \\ - Facharzt \\ - Curriculum
}

Key words

- further training

- microsurgery

- consultant

- curriculum eingereicht $\quad 6.02 .2014$ akzeptiert $\quad 15.05 .2014$

\section{Bibliografie}

DOI http://dx.doi.org/ 10.1055/s-0034-1381996 Handchir Mikrochir Plast Chir 2014; 46: 234-241

(c) Georg Thieme Verlag KG Stuttgart · New York ISSN 0722-1819

Korrespondenzadresse Dr. Jonas Kolbenschlag Klinik für Plastische Chirurgie und Schwerbrandverletzte Handchirurgiezentrum Operatives Referenzzentrum für Gliedmaßentumoren BG Universitätsklinikum Bergmannsheil

Ruhr Universität Bochum Bürkle-de-la Camp Platz 1 44789 Bochum jonaskolbenschlag@ googlemail.com

\section{Zusammenfassung}

$\nabla$

Die rekonstruktive Mikrochirurgie stellt einen essentiellen Bestandteil der Plastischen Chirurgie dar. Um den hohen technischen Anforderungen der heutigen Verfahren gewachsen zu sein bedarf es einer strukturierten mikrochirurgischen Weiterbildung. Strukturierte Curricula zur fundierten Vermittlung dieser Fähigkeiten sind jedoch bisher in Deutschland nicht flächendeckend etabliert. Ziel dieser Umfrage war es daher, die Vorstellungen und Wünsche von Weiterbildungsassistenten und Weiterbildern bezüglich einer optimalen mikrochirurgischen Ausbildung zu erheben. Hier zeigte sich, dass die Erwartungen beider Seiten an ein solches Curriculum sehr nahe bei einander lagen. Dagegen wurden auch Faktoren identifiziert werden, die dessen praktische Umsetzung erschweren, allen voran der zunehmende ökonomische Druck. Basierend auf den erhobenen Daten könnte von Vertretern der Weiterbilder und der Weiterbildungsassistenten aus mikrochirurgisch aktiven Abteilungen unter Einbeziehung nationaler Fachgesellschaften ein „Curriculum Mikrochirurgie“ erstellt werden, das die Mindestanforderungen an eine strukturierte Mikrochirurgische Ausbildung klar definiert. Die praktische Umsetzung eines solchen Curriculums wird jedoch nicht ohne signifikanten Einsatz von Ressourcen zu erreichen sein. Dieser Aufwand erscheint vor dem Hintergrund der kontinuierlichen technischen Innovation, der gesteigerten Patientenansprüche und der aufgrund der regulierten Wochenarbeitszeit limitierten Weiterbildungszeit unbedingt gerechtfertigt.

\section{Einleitung}

$\nabla$

Die rekonstruktive Mikrochirurgie stellt einen essentiellen Bestandteil der Plastischen Chirurgie dar. Durch stetige Weiterentwicklung und Verfeinerung mikrochirurgischer Techniken kön-

\section{Abstract \\ $\nabla$}

Reconstructive microsurgery is an essential part of plastic surgery. To live up to the high technical demands of today's sophisticated techniques, a structured microsurgical training is required. However, such curricula are rare in Germany. We therefore evaluated the concepts and requests of trainers as well as trainees regarding an optimal microsurgical training. We found that the demands of both sides to be fairly similar. However, there were factors potentially hindering the implementation of such curricula, foremost the increasing economic pressure. Based on our findings, representatives of microsurgical trainers and trainees, together with national societies might be able to establish a national curriculum for microsurgical training. The clinical implementation of such a structured training will require significant personal resources. However, this expenditure seems to be justified by the increasing complexity of techniques, the rising demand of patients and the limited time for surgical training. 
schen Weiterbildung in Deutschland im wissenschaftlichen Schrifttum bisher eher unterrepräsentiert behandelt [1,2]. Eine 2009 durchgeführte Umfrage zum Stand der mikrochirurgischen Weiterbildung in Deutschland zeigte, dass ein Großteil der teilnehmenden Weiterbildungsassistenten die Mikrochirurgie zu ihrem späteren Tätigkeitsfeld rechnete. Weniger als einem Drittel der Befragten war es möglich, am Tier oder am Modell zu trainieren. Lediglich 19\% der Befragten gaben an, dass an ihrer Ausbildungseinrichtung ein strukturiertes Curriculum Mikrochirurgie existiert. Auch die direkte klinische Ausbildung war nahezu exklusiv höheren Weiterbildungsabschnitten vorbehalten. So gaben $70 \%$ der Umfrageteilnehmer an, dass freie Lappenplastiken an ihrer Klinik primär durch Oberärzte durchgeführt würden [1].

Dabei zeigen Studien aus allen Bereichen der (Mikro-)Chirurgie dass bereits mit relativ geringem Übungsaufwand eine deutliche Verbesserung mikrochirurgischer Fähigkeiten erreicht werden kann [3-5]. Diese Fähigkeiten können unabhängig vom Alter auch bereits in sehr frühem Weiterbildungsstadium erfolgreich vermittelt werden [3] und es existiert eine Vielzahl an geeigneten Modellen und Simulatoren [6-11]. Im eigenen Patientengut zeigte sich, wie auch in der Literatur [12], dass freie Lappenplastiken, unter Aufsicht erfahrener Mikrochirurgen, durchaus als Weiterbildungseingriff gelten können, ohne dass dies mit einer erhöhten Komplikationsrate einhergeht [13]. Unterstützt wird diese These weiterhin durch Ergebnisse die nahe legen, dass nicht die reine Weiterbildungsauszeit selbst zu besseren Ergebnissen führt, sondern vielmehr die entsprechende Ausbildung der Weiterbildungsassistenten (WBA) [14]. Das eigenständige Erlernen grundlegender mikrochirurgischer Techniken und Fertigkeiten, sogenannter „Skills“, ist an vielen Kliniken möglich und kann durch mikrochirurgische Übungskurse und entsprechende Modelle weiter vertieft werden [6,8]. Sicher besteht auch auf dieser Ebene noch Optimierungsbedarf, allerdings scheint derzeit der strukturierte Transfer der so erlernten Basisfähigkeiten in die klinische Tätigkeit am Patienten die größere Herausforderung zu sein. Ohne die zeitnahe Implementierung mikrochirurgischer Grundkenntnisse in der operativen Tätigkeit ist der Nutzen eines dezidierten mikrochirurgischen Basis-Trainings fraglich.

International gibt es bereits seit einiger Zeit Bestrebungen, die mikrochirurgische Weiterbildung zu strukturieren und ihre Ergebnisse objektiv zu evaluieren [15-21]. Ziel der aktuellen Umfrage war es daher die Vorstellungen einer idealen mikrochirurgischen Ausbildung sowohl bei WBA als auch bei Weiterbildern abzufragen, um so sowohl den Bedarf an einem strukturierten Curriculum in Deutschland zu evaluieren als auch ggf. potentielle Bausteine eines solchen zu identifizieren. Zudem erfolgte die Abfrage von Basiskennzahlen der Institutionen sowie insbesondere die von WBA und Weiterbildern gewünschte Mindesteingriffszahlen.

\section{Material \& Methoden}

Wir führten eine webbasierte Umfrage unter allen 462 assoziierten Mitgliedern der Deutschen Gesellschaft der Plastischen, Rekonstruktiven und Ästhetischen Chirurgen (DGPRÄC) durch. Hierzu wurde ein Fragebogen mit insgesamt 31 Einzelposten erstellt. Dieser wurde, nach freundlicher Zustimmung durch das Präsidium der DGPRÄC, von der Geschäftsstelle der DGPRÄC in Form eines zugangsbeschränkten, elektronischen Fragebogens im Internet zur Verfügung gestellt. Die Einladungen zur Teilnahme wurden von der Geschäftsstelle der DGPRÄC per Email an alle assoziierten Mitglieder der Gesellschaft verschickt.
Zudem wurden von 24 Chefärzten (CÄ) mit mikrochirurgischem Schwerpunkt, die über mehrjährige Erfahrung in der Leitung eines mikrochirurgischen Ausbildungsprogramms verfügen, korrespondierende Fragebögen ausgefüllt. Diese Fragebögen wurden entweder per Email versandt $(n=13)$ oder im Rahmen von Kongressen verteilt und bearbeitet $(n=11)$.

Die ersten 6 der 31 Fragen bezogen sich auf persönliche bzw. institutionelle Kenngrößen und fragten neben dem entsprechenden Weiterbildungsstand und der Versorgungsstufe der jeweiligen Abteilung auch die Frequenz mikrochirurgischer Eingriffe an selbiger sowie den relativen Anteil an freien Lappenplastiken an den genannten Operationen ab. Im Anschluss wurden 7 Fragen zum Themenkomplex „Mikrochirurgie-Kurs“ und Trainingsmöglichkeiten gestellt. Hier wurde u.a. danach gefragt, ob und ggf. wann ein solcher Kurs belegt wurde, ob der WBA bei der Teilnahme unterstützt wurde, ob der Kurs den Einstieg in das mikrochirurgische Arbeiten erleichterte und ob ein solcher Kurs an der jeweiligen Klinik Eingangsvoraussetzung für das mikrochirurgische Arbeiten ist. Zudem wurde erfragt, ob vor Ort Möglichkeiten zum selbständigen Erlernen mikrochirurgischer Basisfertigkeiten vorgehalten werden, ob diese durch den WBA genutzt wurden und ob an der jeweiligen Klinik ein strukturiertes „Curriculum Mikrochirurgie“ im Sinne eines schriftlich fixierten und am Weiterbildungsstand orientieren Weiterbildungskonzepts existiert. Zum Abschluss der Erhebung des Status quo erfolgte die Abfrage bzgl. der Zeitpunkte, ab denen WBA als erste Assistenz bzw. Operateur (unter Aufsicht und selbstständig) fungieren.

Im Anschluss erfolgte die Abfrage persönlicher Präferenzen im Hinblick auf das bevorzugte Teilgebiet der Plastischen Chirurgie, der voraussichtlichen späteren Tätigkeit sowie der Bereitschaft sich für ein mikrochirurgisches Fellowship zu bewerben, bzw. für eine mikrochirurgische Weiterbildungsstelle den Arbeitsplatz zu wechseln. Der folgende Teil beschäftigte sich mit den persönlichen Vorstellungen einer „idealen mikrochirurgischen Weiterbildung“. Um hier eine bestmögliche Präzisierung der Vorstellungen zu erreichen mussten die Umfrageteilnehmer zu jeder Frage eine persönliche Rangliste aus 4 vorgegebenen Antwortmöglichkeiten erstellen. Abschließend wurde zur Konkretisierung der Ergebnisse noch die jeweiligen Vorstellungen bzgl. des optimalem zeitlichen Verlaufs (1. Assistenz, Eingriffe unter Aufsicht bzw. selbstständig) sowie der Mindestanzahl an mikrochirurgischen Eingriffen im Rahmen der Weiterbildung abgefragt. Der vollständige Fragebogen wird gerne auf Anfrage per Email zugesandt. Die Ergebnisse sind als Mittelwerte mit dem jeweiligen Intervall angegeben.

\section{Ergebnisse}

Im Umfragezeitraum vom 15. Juli bis 31. August 2013 nahmen insgesamt 76 der 462 assoziierten Mitglieder teil (16,5\%), 20 der 24 an Chefärzte versandten Bögen wurden vollständig zurückgesandt und konnten ausgewertet werden (83\%).

\section{Basisdaten}

Von den teilnehmenden WBA war die Mehrheit (54\%) an Häusern der Maximalversorgung tätig, 34\% arbeiteten an größeren kommunalen Häusern und Lehrkrankenhäusern, $9 \%$ an Häusern der Grund- und Regelversorgung und 3\% in einer Praxis. Der überwiegende Anteil der befragten Chefärzte führte eine Klinik an einem Haus der Maximalversorgung (64\%), während 24\% 


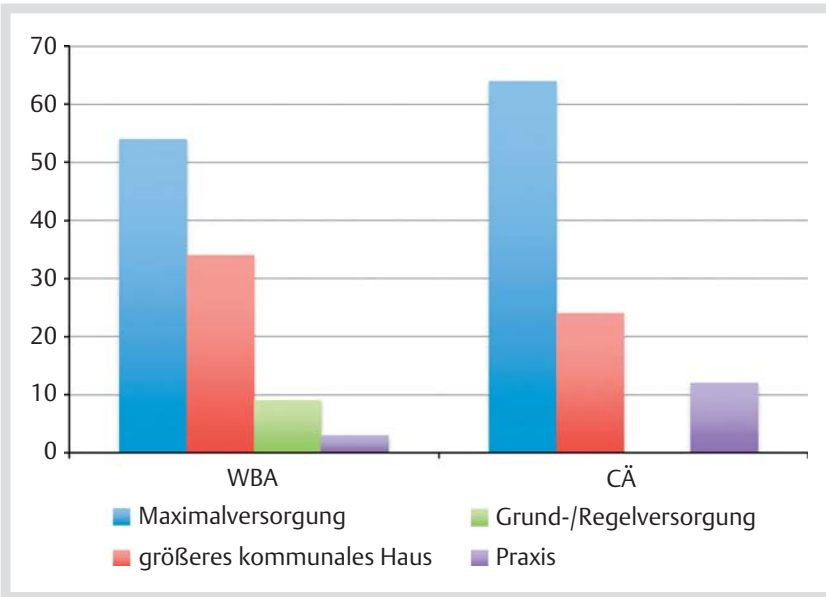

Abb. 1 Verteilung der Versorgungsstufen der jeweiligen Kliniken, aufgeteilt nach WBA und CÄ (in \%).

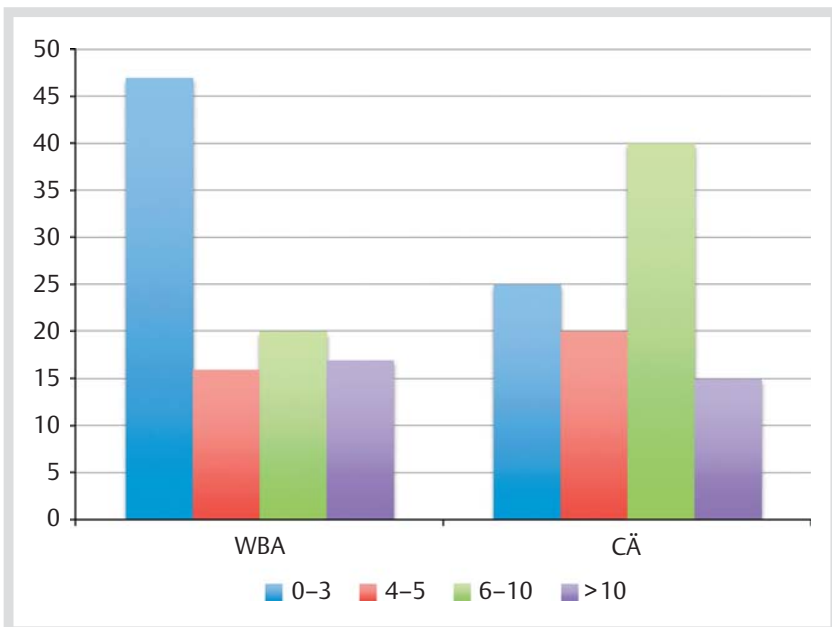

Abb. 2 Gruppierte Angabe der pro Woche in der jeweiligen Klinik durchgeführten mikrochirurgischen Eingriffe.

eine Abteilung an einem größeren kommunalen Haus und Lehrkrankenhaus leiteten und $12 \%$ in eigener Praxis tätig waren ( $\bullet$ Abb. 1). Bezüglich der Anzahl an wöchentlichen mikrochirurgischen Eingriffe (alle Eingriffe unter Nutzung eines Operationsmikroskop, inkl. Nervenkoaptionen und Revaskularisationen) gaben $47 \%$ der WBA an, dass an ihrer Abteilung 0-3 solcher Eingriffe pro Woche durchgeführt wurden. Mehr als 10 durchgeführte Eingriffe pro Woche gaben lediglich $17 \%$ der Teilnehmer an. Bei den befragten CÄ gaben $40 \%$ an, an ihrer Klinik 6-10 mikrochirurgische Eingriffe pro Woche durchzuführen, während 25\% 0-3 Eingriffe berichteten ( $\boldsymbol{A}$ Abb. 2, 3).

\section{Übungsmöglichkeiten/Mikrochirurgie-Kurse}

$48 \%$ der befragten WBA hatten vor Ort Zugriff auf mikrochirurgische Übungsmöglichkeiten, was $73 \%$ auch bereits nutzen. Ein strukturiertes Curriculum Mikrochirurgie existierte in $9 \%$ der Kliniken der WBA und in 20\% der Einrichtungen der befragten CÄ. Sowohl der überwiegende Anteil der WBA (85\%) als auch der CÄ $(80 \%)$ halten ein solches Curriculum für wichtig oder sogar sehr wichtig ( $\bullet$ Abb. 4). Externe mikrochirurgische Weiterbildungsangebote wurden von 59\% der befragten WBA genutzt, im Durchschnitt im 3. Weiterbildungsjahr (1.-6. Jahr).

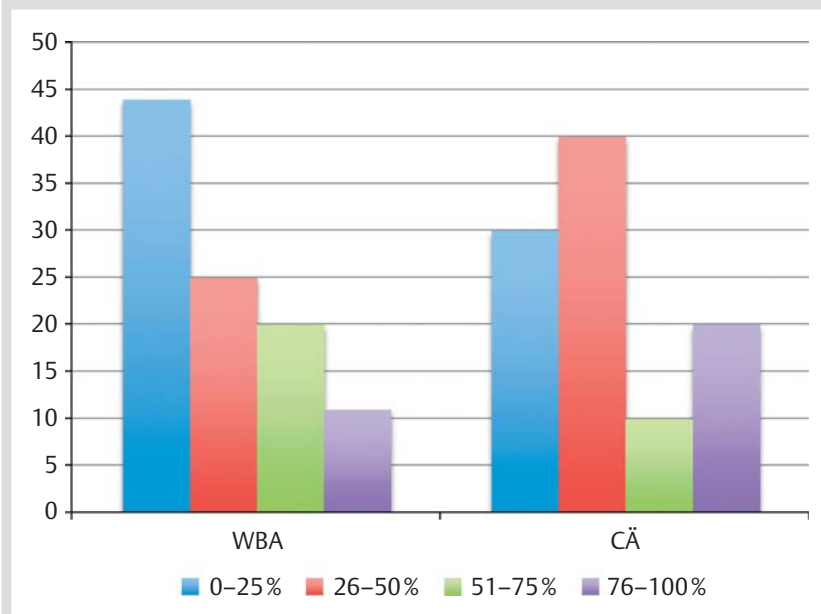

Abb. 3 Anteil der freien Lappenplastiken an allen mikrochirurgischen Eingriffen (in \%).

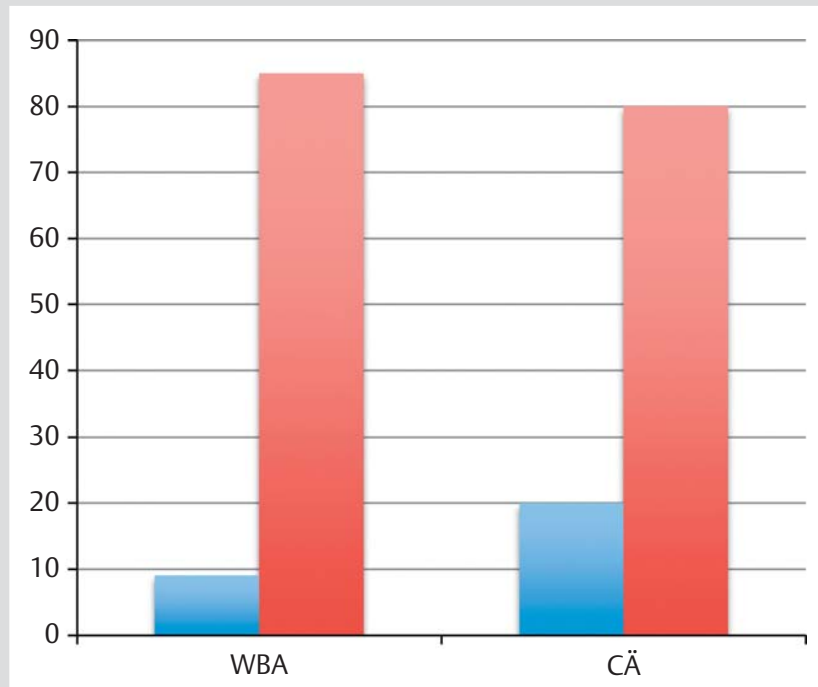

a Curriculum vorhanden $\quad$ Curriculum wichtig / sehr wichtig

Abb. 4 Anteil der WBA/CÄ in deren Klinik ein strukturiertes Curriculum existiert bzw. die ein solches für (sehr) wichtig erachten (in \%).

$42 \%$ der Befragten wurden bei dem Besuch eines Mikrochirurgiekurses von ihrem Arbeitgeber durch Freistellung und/oder Kostenübernahmen unterstützt, 18\% der WBA gaben an, dass an ihrer Klinik ein solcher Kurs Voraussetzung für die klinische Tätigkeit sei. 30\% der WBA hielten einen solchen Kurs für den wichtigsten Punkt vor Beginn der klinischen mikrochirurgischen Tätigkeit, die Überprüfung der eigenen mikrochirurgischen Fähigkeiten durch einen erfahren Ausbilder hingegen wurde von noch mehr WBA als wichtigste Eingangsvoraussetzung angesehen (35\%). Jeweils 50\% der befragen CÄ gaben an, dass eine solche Überprüfung bzw. ein Mikrochirurgiekurs die wichtigsten Vorrausetzungen für mikrochirurgisches Arbeiten darstellen.

\section{Zeitlicher Verlauf}

Als erste Assistenz bei mikrochirurgischen Eingriffen würden WBA laut den CÄ regelhaft ab dem 2. Ausbildungsjahr (Mittelwert 1,8 Jahre, 1-4 Jahre) eingesetzt, die WBA selbst gaben an, 
Tab. 1 Zeitlicher Verlauf der Durchführung erster Assistenzen, Eingriffen unter Aufsicht und selbstständiger Durchführung mikrochirurgischer Eingriffe in Ausbildungsjahren.

\begin{tabular}{|llll} 
& Erste Assistenz & $\begin{array}{l}\text { Eingriffe unter } \\
\text { Aufsicht }\end{array}$ & $\begin{array}{l}\text { selbstständige } \\
\text { Durchführung }\end{array}$ \\
WBA & $2,5(1-6)$ & $3,9(1-9)$ & $5,2(1-10)$ \\
\hline C̈ & $1,8(1-4)$ & $3,2(2-5)$ & $4,6(3-6)$ \\
\hline
\end{tabular}

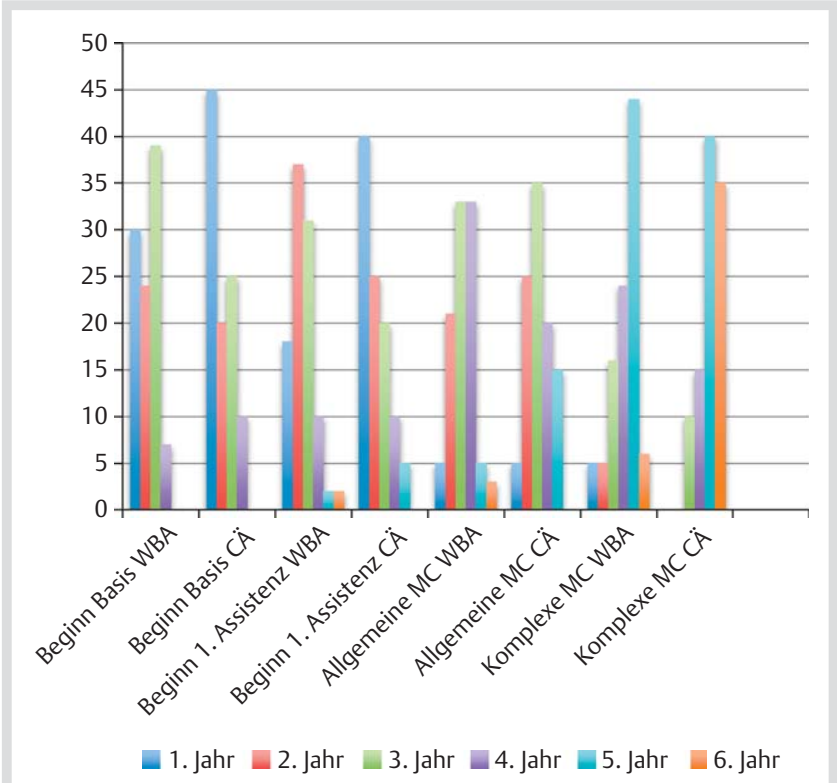

Abb. 5 Vorstellungen von WBA und CÄ bzgl. des Beginns des mikrochirurgischen Basistrainings, der 1. Assistenz bei mikrochirurgischen Eingriffen, der allgemeinen Mikrochirurgie Nerven-/Gefäßnähte) und der komplexen Mikrochirurgie (freie Lappenplastiken/Replantationen) (MC= Mikrochirurgie, WBA= Weiterbildungsassistent, $C \ddot{A}=$ Chefärzte, Angaben in Prozent der Stimmen pro Weiterbildungsjahr).

ab dem 3. Ausbildungsjahr (Mittelwert 2,5 Jahre, 1-6 Jahre) regelhaft als erste Assistenz zu fungieren. Die Durchführung mikrochirurgischer Eingriffe unter Aufsicht erfolgt laut CÄ und WBA im 4. Ausbildungsjahr (3,2 Jahre, 2-5 Jahre bzw. 3,9 Jahre, 1-9 Jahre) während die selbstständige Durchführung solcher Operationen im Mittel im 5. bzw. 6. Jahr der Weiterbildung beginnt (CÄ: 4,6 Jahre, 3-6 Jahre/WBA: 5,2 Jahre, 1-10 Jahre) (• Tab.1).

\section{Die „ideale" mikrochirurgische Ausbildung}

Befragt nach dem idealem Zeitpunkt für den Beginn des mikrochirurgischen Basistrainings gab die Mehrheit der WBA (54\%) die ersten beiden Weiterbildungsjahre an, auch die erste Tätigkeit als 1. Assistenz würde sich die Mehrheit im 2. Ausbildungsjahr wünschen (37\%). Die Mehrheit der befragten CÄ würde sogar einen früheren Beginn des Basistrainings (45\% im 1. Jahr) und der 1. Assistenz (40\% im 1. Jahr) begrüßen. Vonseiten der WBA ist ein Beginn eines allgemeinen mikrochirurgischen Trainings mit selbstständiger Durchführung von Gefäß- und Nervennähten im 3.-4. Weiterbildungsjahr gewünscht (je 33\%) während der Großteil der CÄ (35\%) einen Beginn im 3. Jahr favorisieren würden. Bezüglich der selbstständigen Durchführung von Replantationen und freien Lappenplastiken wünschte sich der überwiegende Anteil (44\%) der Befragten WBA einen Beginn im 5. Ausbildungsjahr, vonseiten der CÄ wurden das 5. und 6. Jahr favorisiert (40 bzw. 35\%) (๑ Abb. 5).
Tab. 2 Vorstellung von WBA und CÄ bzgl. der optimalen Anzahl an 1. Assistenzen bei mikrochirurgischen Eingriffen bzw. derer Durchführung im Rahmen der Facharztausbildung.

\begin{tabular}{|llll} 
& 1. Assistenzen & $\begin{array}{l}\text { Allgemeine } \\
\text { mikrochirurgische } \\
\text { Eingriffe }\end{array}$ & $\begin{array}{l}\text { Komplexe } \\
\text { mikrochirurgische } \\
\text { Eingriffe }\end{array}$ \\
\hline WBA & $55(10-300)$ & $61,2(5-500)$ & $21(0-100)$ \\
\hline CA & $31,3(10-60)$ & $34,4(15-100)$ & $12,8(0-30)$
\end{tabular}

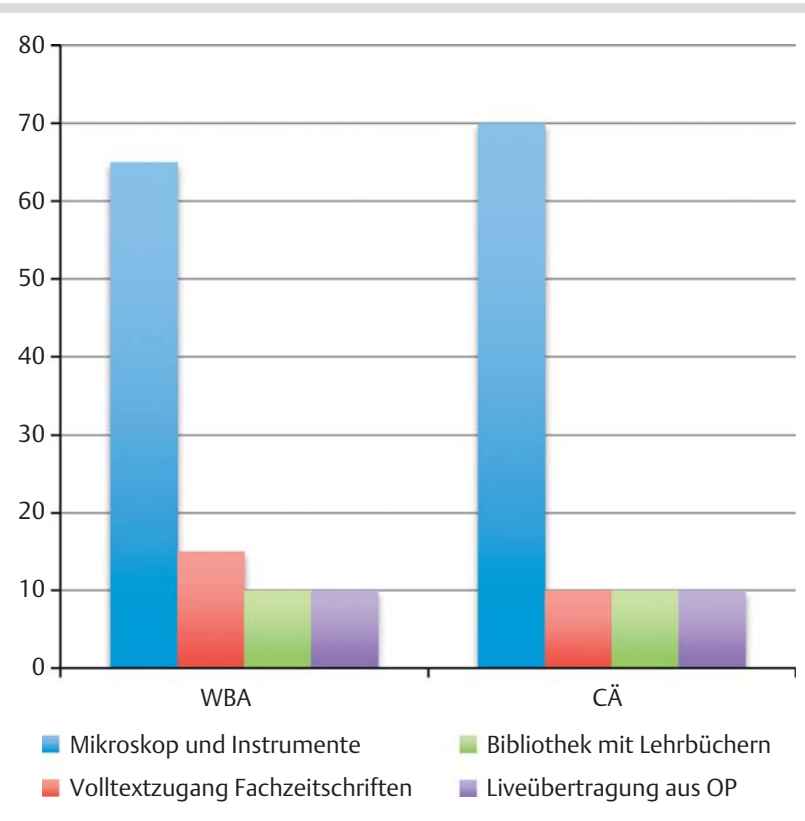

Abb. 6 Verteilung der Forderungen von WBA und CÄ an die optimale Infrastruktur für die mikrochirurgische Weiterbildung (in \%).

Befragt nach den Mindesteingriffszahlen im Rahmen eines idealen Weiterbildungsprogramms forderten die WBA im Mittel 55 erste Assistenzen bei mikrochirurgischen Eingriffen, 61,2 selbstständig durchgeführte allgemeine (Gefäß-/Nervennaht) und 21 komplexe (Replantation, freie Lappenplastik) Eingriffe. Von den CÄ wurden für ein optimales Weiterbildungsprogramm im Mittel 31,3 erste Assistenzen, 34,4 allgemeine und 12,8 komplexe Eingriffe gefordert. Darauf aufbauend forderten die WBA im Mittel 120 allgemeine und 55 komplexe Eingriffe an ihrer Ausbildungsklinik, die CÄ 104 allgemeine und 43 komplexe Eingriffe (० Tab. 2).

Befragt nach den für Sie wichtigsten Aspekten bzgl. der Infrastruktur einer Weiterbildungseinrichtung waren mikrochirurgische Übungsplätze (Zugang zu einem Operationsmikroskop und -besteck sowie die Verfügbarkeit eines entsprechenden Modells) klar auf Platz 1 (70\% der CÄ, 65\% der WBA) ( Abb. 6). Ebenfalls einheitlich waren die Forderungen von WBA und CÄ im Bezug auf die optimale mikrochirurgische Basisweiterbildung. 50\% der WBA und 55\% der CÄ nannten hier die direkte Betreuung durch einen erfahrenen Instruktors als wichtigsten Aspekt ( $\bullet$ Abb. 7). Bezüglich des mikrochirurgischen Weiterbilders selbst nannten sowohl CÄ als auch WBA mit Abstand am häufigsten dessen Erfahrung als wichtigste Eigenschaft ( 80 bzw. $70 \%)$. Am zweithäufigsten wurde das Vertrauen des Ausbilders in den WBA genannt (je 15\%). Die Reputation des Ausbilders wurden weder von den WBA noch von den CÄ in diesem Kontext als relevant eingestuft ( $\bullet$ Abb. 8). 


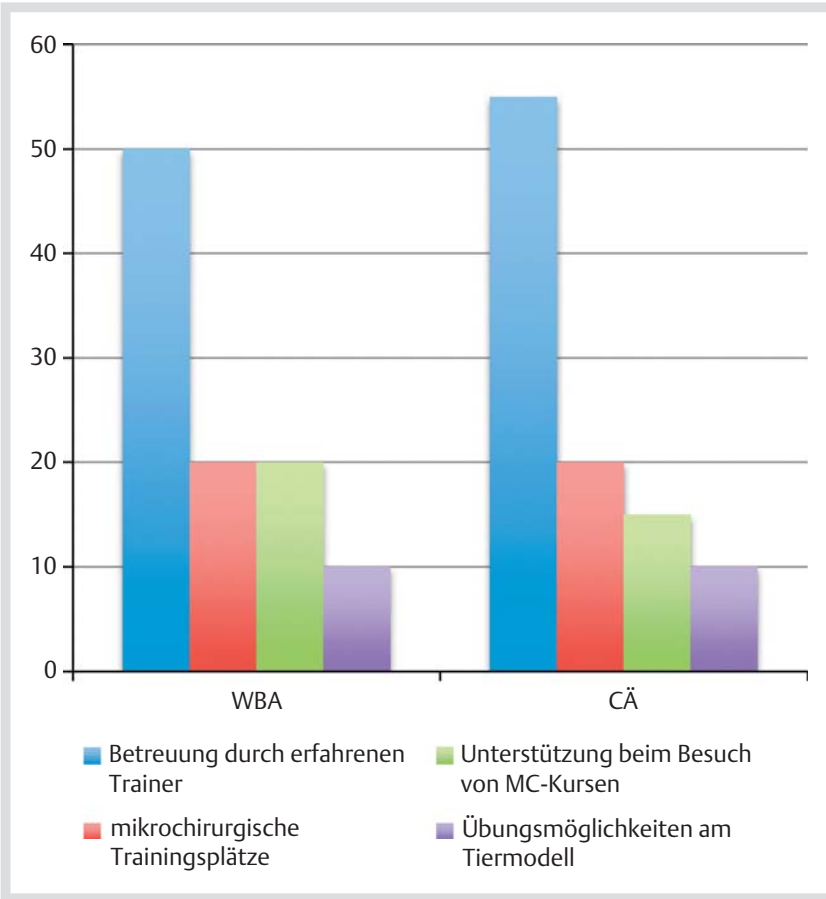

Abb. 7 Verteilung der Forderungen von WBA und CÄ an eine optimale mikrochirurgische Basisausbildung (in \%).

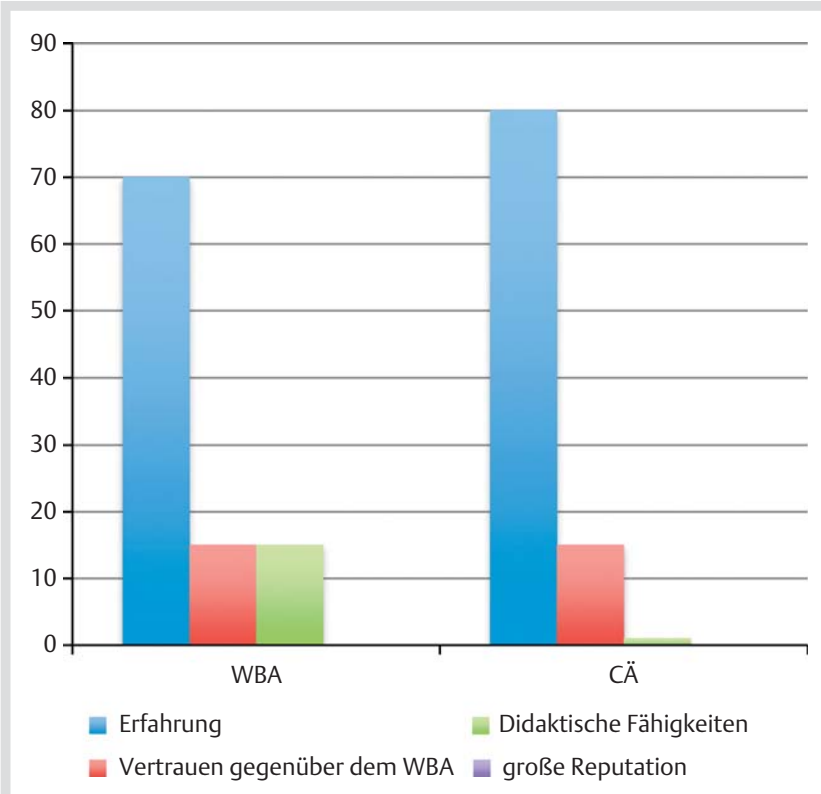

Abb. 8 Verteilung der Forderungen von WBA und CÄ an den mikrochirurgischen Ausbilder (in \%).

Auch bei der Frage nach dem Faktor der die Qualität der mikrochirurgischen Ausbildung am stärksten beeinflusst waren sich WBA und CÄ einig: Die Fallzahlen der Klinik wurden hier als vorrangig genannt ( $50 \%$ der WBA, $40 \%$ der CÄ) (• Abb. 9).

Als wichtigsten Einflussfaktor für eine optimale mikrochirurgische Ausbildung nannten je 35\% der CÄ ein strukturiertes Curriculum bzw. eine höchst mögliche Zahl an Eingriffen. Bei den WBA rangierte interessanterweise die Eingriffszahl noch deutlich vor einem Curriculum ( 45 vs. $35 \%$ ) ( $\bullet$ Abb. 10).

Als größtes Problem für eine hochwertige mikrochirurgische Ausbildung gaben $40 \%$ der CÄ den zunehmenden ökonomischen

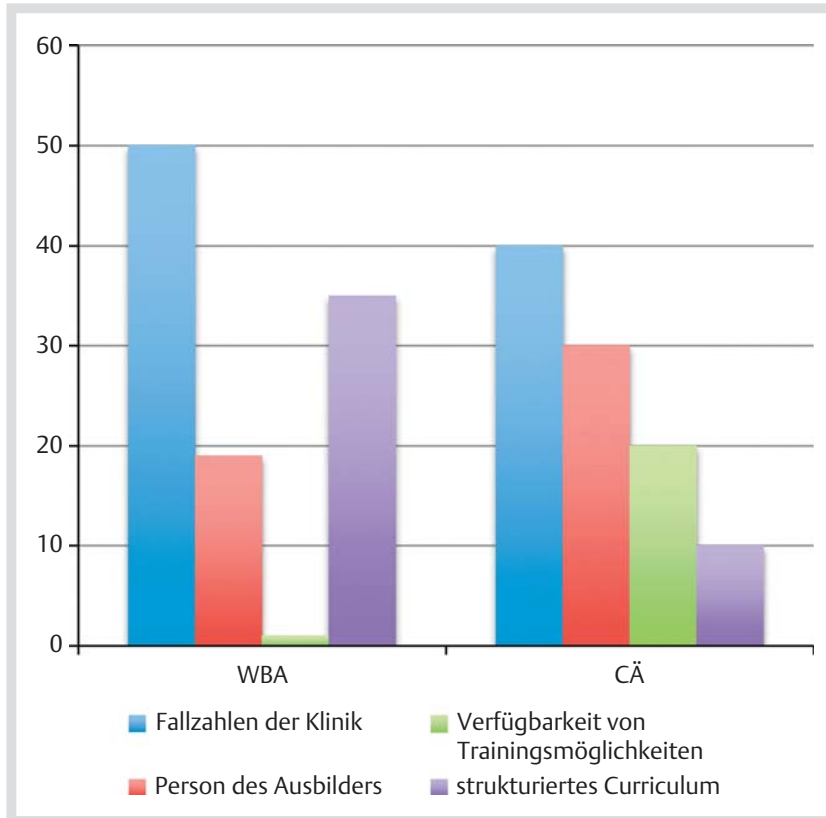

Abb. 9 Verteilung der Aussagen von WBA und CÄ wovon die Qualität einer mikrochirurgischen Ausbildung am stärksten abhängt (in \%).

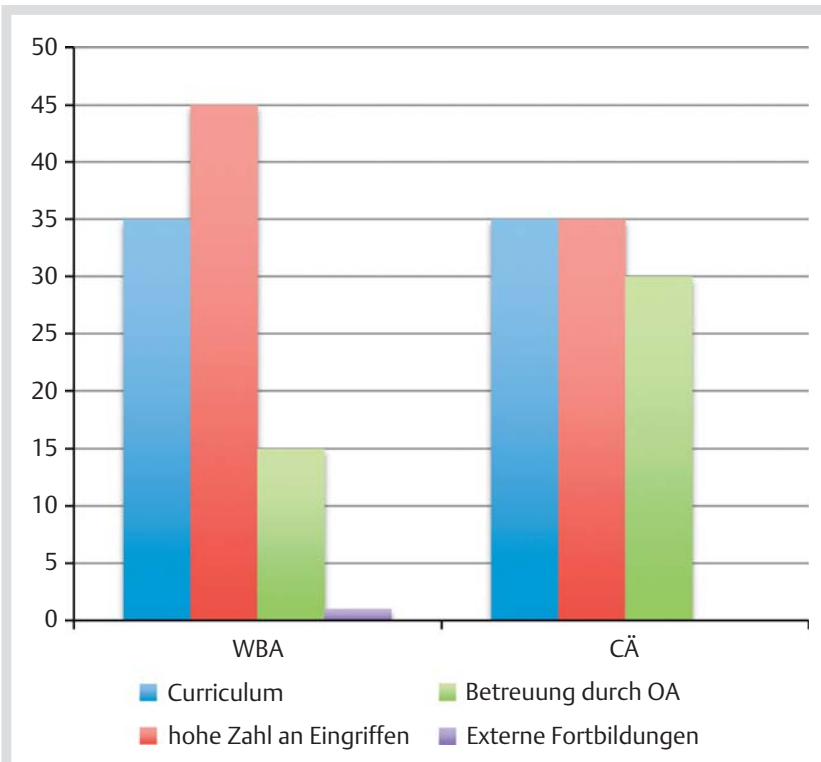

Abb. 10 Verteilung der Forderungen von WBA und CÄ an eine optimale mikrochirurgische Ausbildung (in \%).

Druck und den damit verbunden Zwang zur Optimierung der OP-Zeiten an. 20\% der Befragten Weiterbildungsassistenten sahen eine zu geringe Zahl an Ausbildungseingriffen als Hauptproblem an.

\section{Diskussion}

Im Vergleich zu der Umfrage von Daigeler et al. aus dem Jahre 2009 zeigten sich keine signifikanten Veränderungen bezüglich der strukturellen Voraussetzungen [1]. Weiterhin sind die grundlegen Voraussetzungen zum Erlernen mikrochirurgischer Techniken an einem großen Teil der Kliniken verfügbar und werden auch genutzt. Bezüglich des Transfers dieser Basisfähigkei- 
ten in die Klinik herrscht allerdings Uneinigkeit. So sind zwar Großteile der befragten WBA und CÄ der Meinung, dass ein strukturiertes Curriculum für die mikrochirurgische Ausbildung sehr wichtig wäre. Umgesetzt werden derartige Konzepte jedoch nach wie vor nur in einem kleinen Teil der Kliniken [22].

Vor der Entwicklung eines strukturierten Curriculums für die mikrochirurgische Ausbildung müssen unbedingt die Wünsche und Vorstellungen von Weiterzubildenden und Weiterbildnern eruiert werden. Erfreulicherweise zeigt diese Studie erstmals, dass die Vorstellungen von WBA und CÄ bezüglich einer idealen mikrochirurgischen Ausbildung in Deutschland recht nahe beieinander liegen. Die Erstellung eines für alle Seiten zufriedenstellenden Curriculums scheint daher ein realistisches Unterfangen. Dessen praktische Umsetzung bedarf jedoch auch der engen Einbindung nicht-medizinischer Führungskräfte in den Krankenhäusern, welche von der Notwendigkeit der Umsetzung vor dem Hintergrund der Konkurrenzfähigkeit und Qualitätssicherung überzeugt werden müssen. Insbesondere die zunehmenden ökonomischen Zwänge und die resultierenden Forderung nach möglichst kurzen OP-Zeiten und maximaler Effizienz sehen viele Weiterbilder als Bedrohung für die mikrochirurgische Ausbildung. Zudem erfordert die Umsetzung eines strukturierten Curriculums in der Mikrochirurgie signifikante personelle und finanzielle Ressourcen, die vor dem Hintergrund des Kostendrucks im Deutschen Gesundheitssystem mit weitestgehend ausgeschöpften Effizienzreserven in Ausbildungskliniken schwer mobilisierbar sind. Dabei setzt insbesondere die sowohl von WBA als auch von CÄ als wichtiger Punkt genannte intensive Betreuung durch einen erfahrenen Weiterbilder eine ausreichende Personaldecke voraus. Häufig sind gerade die mikrochirurgisch erfahrenen Fach- und Oberärzte aufgrund der stetigen Leistungsverdichtung derartig belastet, dass auch hier nicht ausreichend Zeit für die Weiterbildung zur Verfügung steht.

Darüber besteht die Sorge, dass die intraoperative Weiterbildung zu verlängerten OP-Zeiten führen könnte, was wiederum zu direkten und indirekten Mehrkosten führt und die erfahrenen Fach- und Oberärzte potentiell länger bindet [23,24]. Zwar kann insbesondere bei freien Lappenplastiken einer möglichen Verlängerung der OP-Zeit bspw. durch den Einsatz zweier Operations-Teams entgegen gewirkt werden, dies setzt jedoch auch eine entsprechende Personaldecke voraus.

Im Zusammenspiel mit der mangelnden Abbildung der Weiterbildung im DRG-Vergütungssystem wird also kein finanzieller Anreiz geboten, solche Systeme zu etablieren [25,26]. Die zunehmende Leistungsverdichtung bewirkt vielmehr gegenteilige Effekte. Inzwischen stellen einige Kliniken aus ökonomischen Gründen bevorzugt Fachärzte ein, die keinerlei Weiterbildung bedürfen und flexibel und unabhängig eingesetzt werden können. Neben monetären Aspekten wirkt in vielen anderen chirurgischen Disziplinen auch die schwache Bewerberlage als Antrieb zur Veränderung und Verbesserung der Aus- und Weiterbildung. Da sich für das Fach „Plastische und Ästhetische Chirurgie“ jedoch weiterhin deutlich mehr Bewerber als freie Stellen finden, besteht in dieser Disziplin vor dem Hintergrund der Personalrekrutierung vordergründig kein akuter Handlungsbedarf. Hierbei sollte allerdings berücksichtigt werden, dass insbesondere hochmotivierte und hochbegabte Bewerber (sog. „high potentials“) ihre Ausbildungsklinik sehr bewusst wählen und dass daher ein gut strukturiertes mikrochirurgisches Ausbildungsprogramm ein klarer Wettbewerbsvorteil im Kampf um die besten Mitarbeiter sein kann. Vor allem aber darf die moralische Ver- pflichtung zur Aus- und Weiterbildung des ärztlichen Nachwuchses bei allem ökonomischen Druck nicht ignoriert werden, wie es bereits in einer der grundlegendsten ärztlichen Statuten, der Deklaration von Genf, gefordert ist [27].

Eine vor dem Hintergrund der endlichen Fallzahlen und limitierten Weiterbildungskapazität relevante Frage ist zudem, inwiefern die fortgeschrittene rekonstruktive Mikrochirurgie im gleichen Maß an jeden Weiterbildungsassistenten vermittelt werden kann. Zwar zeigte sich in unserer Umfrage ein großes Interesse an diesem Feld, jedoch ist hier aufgrund des Themas der Umfrage auch von einem gewissen Bias auszugehen. In Anbetracht der teilweise sehr hohen Zahl an geforderten Ausbildungseingriffen pro Person in Relation zu den auch an großen Ausbildungszentren tatsächlich durchgeführten mikrochirurgischen Operationen sind mikrochirurgische Ausbildungseingriffe als rares Gut zu betrachten. Es bedarf daher unbedingt einer ehrlichen Selbsteinschätzung aller Beteiligten um diese Weiterbildungseingriffe bestmöglich im Sinne einer strukturierten Weiterbildung zu verteilen.

Dies ist insbesondere auch vor dem Hintergrund der anstehenden Novellierung der Musterweiterbildungsordnung zum Facharzt für Plastische und Ästhetische Chirurgie von Bedeutung. Zwar sind aktuell rekonstruktive Eingriffe einschließlich mikrochirurgischer Techniken gefordert, eine konkrete Anzahl an solchen Techniken wird jedoch nicht angegeben. Vielmehr können die pro Körperregion vorausgesetzten rekonstruktiven Eingriffe auch bspw. durch lokale Lappenplastiken erbracht werden. Dies ermöglicht zwar eine flexiblere Auslegung der geforderten Ausbildungsinhalte und damit eine bessere Anpassung an die lokalen Ausbildungsverhältnisse, gleichzeitig aber auch das potentielle Erlangen des Facharztes für Plastische und Ästhetische Chirurgie ohne jedwede Erfahrung in der Mikrochirurgie [28]. Im Rahmen der Novellierung der Musterweiterbildungsordnung kann hier gegebenenfalls durch die Einführung von Mindestzahlen für mikrochirurgische Eingriffe eine gemeinsame Grundlage geschaffen werden. Hiervon ausgehend könnte dann auch ein gewisser Ausbildungsanspruch abgeleitet werden könnte. Bei der Festlegung solcher konkreter Zahlen ist jedoch unbedingt die, unter anderen in dieser Umfrage dargestellte, Versorgungsrealität in Deutschland zu berücksichtigen, um realistische Weiterbildungsziele zu gewährleisten.

In Anlehnung an das amerikanische Ausbildungssystem wäre auch die Einrichtung von „fellowship“-Programmen zur vertieften mikrochirurgischen Ausbildung nach Erlangung der Facharztqualifikation ein denkbarer Weg, um eine zielgerichtete und fundierte mikrochirurgische Ausbildung zu gewährleisten.

Grundsätzlich manifestiert sich am Komplex der Mikrochirurgie aber das Problem der Weiterbildung als speziellem Deutschen Postgraduierten-Spezifikum: Die Weiterbildung und alle darin enthaltenen Elemente werden als kontinuierliche Qualifizierung, im tariflichen Beschäftigungsverhältnis, aber nicht als spezialisierter Ausbildungsabschnitt bewertet. Damit ist weder eine spezielle Finanzierung innerhalb der DRG-Kostenstruktur, noch ein Ausbildungsanspruch gegeben.

\section{Schlussfolgerung}

Die vorliegende Umfrage zeigt deutlich, dass in Deutschland derzeit sowohl die Voraussetzungen zum Erlenen mikrochirurgischer Basisfertigkeiten gegeben, als auch die Vorstellungen der 
Auszubildenden und Ausbilder bezüglich eines strukturierten mikrochirurgischen Curriculums weitestgehend kongruent sind. Der Entwurf eines „nationalen“ Curriculums zur strukturierten Weiterbildung in der Mikrochirurgie, welches die landesspezifischen Aspekte und Herausforderungen adäquat berücksichtigt, ist daher eine realistische und wünschenswerte Perspektive, die allerdings die enge Zusammenarbeit von Vertretern der WBA und der Weiterbilder erfordert. Vonseiten der deutschsprachigen Arbeitsgemeinschaft für Mikrochirurgie der peripheren Nerven und Gefäße (DAM) gibt es hierzu bereits Vorarbeiten, ebenso wie zur Zertifizierung mikrochirurgischer Kurse [1,2]. Solche, mit einer Prüfung endenden Kurse, erlauben das strukturierte Erlenen von Basisfähigkeiten am Modell und erfüllen gleichzeitig die von den WBA geforderte Überprüfung der eigenen Fähigkeiten. Ein derartiges Basiszertifikat könnte dann im Rahmen eines „Curriculums Mikrochirurgie“ in die Klinik transferiert und durch entsprechende Eingriffszahl erweitert werden. Die Umsetzung derartiger Konzepte wird jedoch nicht ohne signifikanten Einsatz von Ressourcen erfolgen können. Es obliegt daher aktuell den einzelnen mikrochirurgisch aktiven Einheiten, mikrochirurgische Ausbildungsprogramme zu etablieren. Durch die nationalen Interessensvertretungen bzw. Fachgesellschaften könnten flankierend Grundlagen geschaffen und auf politischer Ebene auf eine Verbesserung der Vergütung der mikrochirurgischen Ausbildung hingewirkt werden. Nur so kann langfristig die Exzellenz der rekonstruktiven Mikrochirurgie und damit auch der Plastischen und Handchirurgie in Deutschland zu Wohle der Patienten erhalten und gefördert werden.

\section{Danksagung}

Wir danken allen Umfrageteilnehmern sowie dem Präsidium und der Geschäftsstelle der DGPRÄC, insbesondere Herrn Strömsdörfer, für die Unterstützung bei der Durchführung der Umfrage und ihrer Auswertung.

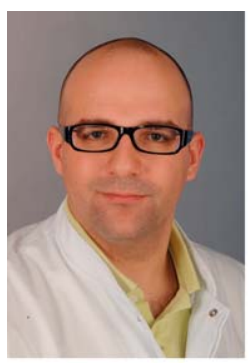

Jonas Kolbenschlag

- 2003-2010 Studium der Humanmedizin an den Universitäten Regensburg, Heidelberg und Bern (PJ)

- 11/2010 Approbation als Arzt an der Universität Heidelberg - 12/2010-08/2012 Assistenzarzt, Klinik für Hand-, Plastische und Rekonstruktive Chirurgie, Schwerbrandverletztenzent-

rum, BG Klinik Ludwigshafen, Klinik für Plastische und Handchirurgie der Ruprecht-Karls-Universität Heidelberg, Direktor: Prof. Dr. med. M. Lehnhardt - seit 09/2012 Assistenzarzt, Universitätsklinik für Plastische Chirurgie und Schwerbrandverletzte, Handchirurgiezentrum, Operatives Referenzzentrum für Gliedmaßentumoren, Berufsgenossenschaftliches Universitätsklinikum Bergmannsheil, Bochum, Direktor: Prof. Dr. med. M. Lehnhardt

\section{Institute}

1 Klinik für Plastische Chirurgie und Schwerbrandverletzte, Handchirurgiezentrum, Operatives Referenzzentrum für Gliedmaßentumoren, BG Universitätsklinikum Bergmannsheil Bochum

2 Sektion für Plastische Chirurgie, Handchirurgie, Intensivstation für Schwerbrandverletzte Universitätsklinikum Schleswig-Holstein, Lübeck

${ }^{3}$ Klinik für Hand-, Plastische und Rekonstruktive Chirurgie, Schwerbrandverletztenzentrum BG-Unfallklinik Ludwigshafen, Klinik für Plastische Chirurgie der Ruprecht-Karls-Universität Heidelberg

${ }^{4}$ Klinik für Plastische, Hand- und Wiederherstellungschirurgie, Zentrum für

Schwerbrandverletzte Medizinische Hochschule Hannover

${ }^{5}$ Plastisch- und Handchirurgische Klinik, Universitätsklinikum Erlangen

\section{Literatur}

1 Daigeler A, Kaempfen A, Beier JP et al. Mikrochirurgische Aus- und Weiterbildung - Bericht des Consensus-Workshops im Rahmen der 31. Jahrestagung der Deutschsprachigen Gemeinschaft für Mikrochirurgie der peripheren Nerven und Gefäße 2009 in Erlangen. Handchir Mikrochir Plast Chir 2010; 42: 273-276

2 Kaempfen A, Daigeler A, Largo RD et al. Bericht zum Workshop und Consensusmeeting zur Mikrochirurgischen Ausbildung an der 32ten Jahrestagung der Deutschsprachigen Arbeitsgemeinschaft für Mikrochirurgie der peripheren Nerven und Gefäße (DAM) 2010 in Basel. Handchir Mikrochir Plast Chir 2011; 43: 262-265

3 Mucke T, Borgmann A, Ritschl LM et al. Microvascular training of medical students and surgeons - A comparative prospective study. J Craniomaxillofac Surg 2013; 41: e187-190

4 Risucci D, Geiss A, Gellman L et al. Surgeon-specific factors in the acquisition of laparoscopic surgical skills. Am J Surg 2001; 181: 289-293

5 Saleh GM, Voyatzis G, Hance J et al. Evaluating surgical dexterity during corneal suturing. Arch Ophthalmol 2006; 124: 1263-1266

6 Colebunders B, Matthew MK, Thomson JG. The use of a surgical glove in microsurgical training: a new point of view. Microsurgery 2010; 30: 505-506

7 Leclere FM, Trelles $M$, Lewbart GA et al. Is there good simulation basic training for end-to-side vascular microanastomoses? Aesthetic Plast Surg 2013; 37: 454-458

8 Nam SM, Shin HS, Kim YB et al. Microsurgical training with porcine thigh infusion model. J Reconstr Microsurg 2013; 29: 303-306

9 Phoon AF, Gumley GJ, Rtshiladze MA. Microsurgical training using a pulsatile membrane pump and chicken thigh: a new, realistic, practical, nonliving educational model. Plast Reconstr Surg 2010; 126: $278 \mathrm{e}-279 \mathrm{e}$

10 Rosen JM, Long SA, McGrath DM et al. Simulation in plastic surgery training and education: the path forward. Plast Reconstr Surg 2009; 123: 729-738 discussion 739-740

11 Sener S, Menovsky T, Maas AI. Use of bubble wrap for microsurgical training. J Reconstr Microsurg 2013; 29: 635-636

12 Patel UA, Lin AC. Flap outcomes when training residents in microvascular anastomosis in the head and neck. Am J Otolaryngol 2013; 34: 407-410

13 Hirche C, Xiong L, Ringwald F et al. Ist eine mikrovaskuläre Lappenplastik als Weiterbildungseingriff geeignet? Eine retrospektive Kohortenstudie mit 391 Patienten. In, 35 Jahrestagung der Deutschsprachigen Arbeitsgemeinschaft für Mikrochirurgie der peripheren Nerven und Gefäße (DAM). 2013

14 Kreymerman P, Silverman AL, Rebecca AM et al. Contradicting an established mantra: microsurgeon experience determines free flap outcome. Plast Reconstr Surg 2012; 130: 507e-512e

15 Balasundaram I, Aggarwal R, Darzi LA. Development of a training curriculum for microsurgery. Br J Oral Maxillofac Surg 2010; 48: 598-606

16 Ghanem AM, Hachach-Haram N, Leung CC et al. A systematic review of evidence for education and training interventions in microsurgery. Arch Plast Surg 2013; 40: 312-319

17 Leung CC, Ghanem AM, Tos $P$ et al. Towards a global understanding and standardisation of education and training in microsurgery. Arch Plast Surg 2013; 40: 304-311

18 Satterwhite T, Son J, Carey J et al. Microsurgery education in residency training: validating an online curriculum. Ann Plast Surg 2012; 68: 410-414

19 Selber JC, Chang EI, Liu J et al. Tracking the learning curve in microsurgical skill acquisition. Plast Reconstr Surg 2012; 130: 550e-557e

20 Temple CL, Ross DC. A new, validated instrument to evaluate competency in microsurgery: the University of Western Ontario Microsurgical Skills Acquisition/Assessment instrument [outcomes article]. Plast Reconstr Surg 2011; 127: 215-222 
21 Chan W, Niranjan N, Ramakrishnan V. Structured assessment of microsurgery skills in the clinical setting. J Plast Reconstr Aesthet Surg 2010; 63: 1329-1334

22 Horch RE, Vogt PM, Schaller HE et al. Strategien zur Sicherung des wissenschaftlichen Nachwuchses in der Plastischen Chirurgie - Eine Standortbestimmung und Zukunftsaussichten. Handchir Mikrochir Plast Chir 2013; 45: 193-199

23 Sasor SE, Flores RL, Wooden WA et al. The cost of intraoperative plastic surgery education. Journal of surgical education 2013; 70: 655-659

24 Castillo A, Zarak A, Kozol RA. Does a new surgical residency program increase operating room times? Journal of surgical education 2013; 70: 700-702
25 Gobel P, Piesche K, Randau T et al. Kostet operative Ausbildung Geld? Eine kontrollierte gesundheitsökonomische Studie auf Grundlage des deutschen DRG-Systems am Beispiel der primären Knieendoprothetik. Zeitschrift für Orthopadie und Unfallchirurgie 2013; 151: 189-193

26 Schroder W, Krones CJ. Die chirurgische Weiterbildung hat ihren Preis. Zentralbl Chir 2012; 137: 144-148

27 Weltärztebund.1948; http://www.bundesaerztekammer.de/downloads/ Genf.pdf

28 Bundesärztekammer. (Muster-)Logbuch über die Facharztweiterbildung Plastische und Ästhetische Chirurgie http://www.bundesaerz tekammer.de/downloads/MLogbuch-7.6-FA_Plastische_Aesthetische Chirurgie.pdf 